\title{
The Questionable Existence of Gravitomagnetic Force
}

\author{
Jaroslav Hynecek ${ }^{1}$ \\ ${ }^{1}$ Isetex, Inc., 905 Pampa Drive, Allen, TX, USA \\ Correspondence: Jaroslav Hynecek, Isetex, Inc., 905 Pampa Drive, Allen, TX 75013, USA. E-mail: \\ jhynecek@netscape.net
}

Received: August 21, 2012 Accepted: September 10, 2012 Online Published: January 5, 2013

doi:10.5539/apr.v5n1p28

URL: http://dx.doi.org/10.5539/apr.v5n1p28

\begin{abstract}
Using a simple model of moving massive parallel plates this paper explains that there cannot be any gravitomagnetic force contrary to a widely spread belief that such a force exists. The existence of this force is usually supported by the analogy with the Maxwell theory of Electromagnetic fields. The gravitomagnetic force, analogous to a force described by the Lorentz force equation, and the accompanied gravitoelectromagnetic field equations, are all derived from Einstein field equations by linearization for the weak gravitational field. The derivation presented in this paper offers a clear reason for the nonexistence of the gravitomagnetic field and therefore also questions the validity of Einstein field equations.
\end{abstract}

Keywords: gravitomagnetic force, Einstein field equations, Maxwell field equations, Lorentz force equation, field energy of charged parallel plates, field energy of massive parallel plates, potential field energy, kinetic field energy

\section{Introduction}

There have been and constantly are many papers published deriving the gravitational field equations for the weak fields by linearization of Einstein field equations. This activity is so popular that it almost seems to be a new branch of physics called the gravitoelectromagnetic (GEM) field theory. An example of already a decade old publication on this topic is the paper authored by Ruggiero and Tartaglia (2002). Another review of the concept was published earlier by Mashhoon, Gronwald, and Lichtenegger (1999). A more sophisticated version of the derivation was published by Clark and Tucker (2000), and even NASA (2004), which has dedicated a significant effort and resources to the detection of gravitomagnetic force, has a webpage on this subject.

The result of linearization of Einstein field equations resembles the Maxwell's equations of the Electro-Magnetic (EM) field theory and from this derivation also follows the analogous equation for the Lorentz force acting on a massive body moving in a gravitational field. The Maxwell-like equations are linear vector equations and are thus easier to handle than the nonlinear tensor equations of the general relativity theory.

In this paper simple models of the two charged parallel plates and the two massive parallel plates are investigated and the field energy resulting from the force acting between the plates is analyzed and compared. The study starts first with the familiar derivation of the EM force acting between the two charged plates when they are at rest relative to the laboratory coordinate system and continues with the case when the plates are moving. Similarly, the field energy resulting from the gravitational force acting between the two moving massive plates is derived using the gravitoelectromagnetic field approach. An advantage of using this arrangement of massive plates is that the curved space-time of the general relativity theory is only outside of the plates, not between them, and thus does not have to be considered for the field energy calculations. This considerably simplifies the analysis, which will clearly show that the gravitomagnetism leads to an unreasonable result and that there can be no gravitomagnetic analogy to the magnetic force and the magnetic field energy of the Maxwell's EM field theory.

\section{Lorentz Force Acting between the Two Parallel Charged Plates and the Corresponding Field Energy}

The attractive force observed between the two charged nonconductive moving plates that have an uniformly distributed embedded charge throughout their thickness can be calculated by considering that in addition to the electrostatic force attracting the plates there is also a force based on the Biot-Savart law acting between the currents, which the moving plates also represent. To calculate this force it is useful to first find the magnetic field intensity $H$ existing in the space between the plates. For the selected configuration the simplest way is to use the integral form of Maxwell's equation for the magnetic field intensity: 


$$
\oint \vec{H} \cdot d \vec{s}=I
$$

The integration path and the current flow are illustrated in a drawing in Figure 1. For the plates with the large width and length in comparison to their spacing the peripheral field effects can be neglected and the magnetic field intensity between the plates up to their internal surfaces written as:

$$
H=\frac{Q}{A} v
$$

Here the $v$ is the plates' velocity, $Q$ the total plates' charge, and $A=L \cdot W$ is the plates' area. Similarly for the electric field intensity from the Gauss law it is:

$$
\oiint \vec{D} \cdot d \vec{S}=Q
$$

where the integrating surface $S$ encloses one of the plates. The electric field intensity $E$ is thus equal to:

$$
E=\frac{Q}{\varepsilon_{0} A}
$$

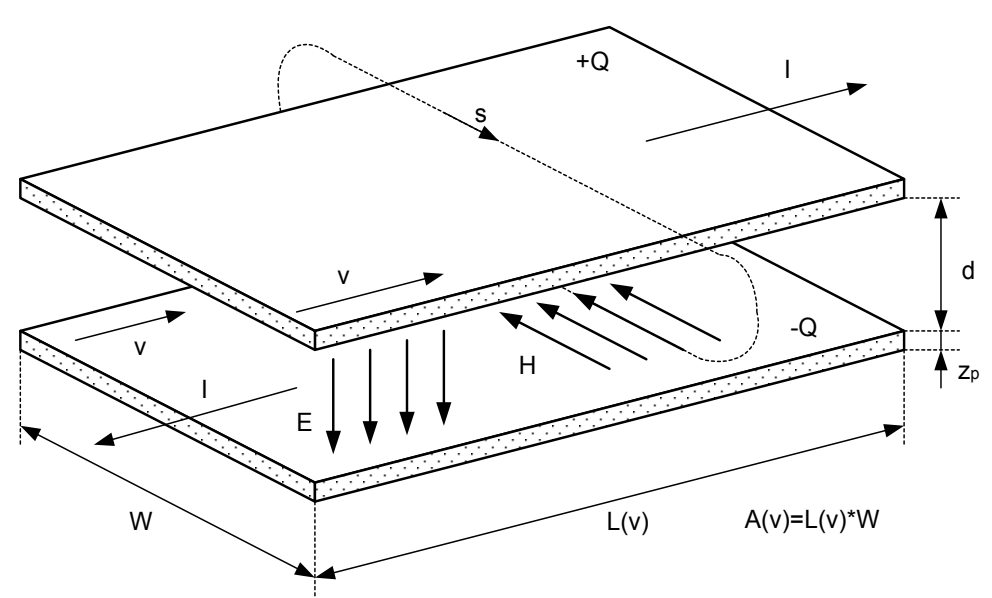

Figure 1. Orientation of current $I$ generated by the moving charged plates and the orientations of the resulting electric and magnetic fields. The magnetic field integration path $s$ used in Equation 1 is also indicated

Both, the magnetic field as well as the electric field intensities are, of course, zero on the external surfaces of the plates, but throughout the plates' thicknesses increase linearly from zero to the full value found between the plates. This is the consequence of the original assumption that the embedded charge distribution within the each plate's volume is uniform. The formula for the force is obtained from the well known Lorentz force equation:

$$
\vec{F}=Q(\vec{E}+\vec{v} \times \vec{B})
$$

which must be integrated over the plate's thickness $z_{p}$.

$$
|\vec{F}|=\frac{Q}{\varepsilon_{0} A}\left(1-\frac{v^{2}}{c^{2}}\right) \int_{0}^{z_{p}} \frac{z}{z_{p}} \frac{Q}{z_{p}} d z
$$

After completion of integration in Equation 6, where $c$ is the speed of light and where the substitution for the parameters: $\vec{D}=\varepsilon_{0} \vec{E}, \vec{B}=\mu_{0} \vec{H}$, and $\varepsilon_{0} \mu_{0}=1 / c^{2}$ was made, the result becomes:

$$
F_{q}=\frac{Q^{2}}{2 \varepsilon_{0} A}\left(1-\frac{v^{2}}{c^{2}}\right)
$$

When the plates are stationary it is $v=0$ and the force equation simplifies as follows: 


$$
F_{q 0}=\frac{Q^{2}}{2 \varepsilon_{0} A_{0}}
$$

The parameters with the subscript zero indicate the values at rest. However, for the moving plates it is necessary to also consider the Lorentz length contraction of the plates' area $A=A_{0}\left(1-v^{2} / c^{2}\right)^{1 / 2}$, which results in the final formula for the force between the moving plates as follows:

$$
F_{q}=\frac{Q^{2}}{2 \varepsilon_{0} A_{0}} \sqrt{1-\frac{v^{2}}{c^{2}}}
$$

In the next step it will be important to find the energy stored in the field when the moving plates are displaced apart at a distance $d$. This is obtained by integrating the force over the distance between the plates leading to the result:

$$
W_{e}=\frac{Q^{2} d_{0}}{2 \varepsilon_{0} A_{0}} \sqrt{1-\frac{v^{2}}{c^{2}}}=m_{0} c^{2} \sqrt{1-\frac{v^{2}}{c^{2}}}
$$

where the following simplifying substitution was made:

$$
\frac{Q^{2} d_{0}}{2 \varepsilon_{0} A_{0}}=m_{0} c^{2}
$$

The parameter $m_{0}$ can be thought of as an equivalent rest mass of the electric field energy. This formula also follows from the well known equation for the energy stored in a capacitor: $W_{c}=1 / 2 Q^{2} / C$, where $C=\varepsilon_{0} A_{0} / d_{0}$ is the plates' capacitance. However, this is not all the energy when the setup is moving. This is only the potential energy. There is also an energy stored in the magnetic field that was not taken into account even though the force on the plates resulting from the currents has been included. The field magnetic energy is calculated as follows:

$$
W_{m}=\frac{1}{2} \mu_{0} H^{2} d_{0} A=\frac{Q^{2} d_{0}}{2 \varepsilon_{0} A_{0}} \frac{v^{2} / c^{2}}{\sqrt{1-v^{2} / c^{2}}}=\frac{m_{0} v^{2}}{\sqrt{1-v^{2} / c^{2}}}
$$

By combining these two results the total EM field energy stored by the moving plates that move in the parallel direction to their surfaces is thus as follows:

$$
W_{e m \|}=V_{\|}+T_{\|}=m_{0} c^{2} \sqrt{1-v^{2} / c^{2}}+\frac{m_{0} v^{2}}{\sqrt{1-v^{2} / c^{2}}}=\frac{m_{0} c^{2}}{\sqrt{1-v^{2} / c^{2}}}
$$

The total EM field energy thus consists of the two components; the field potential energy:

$$
V_{\|}=m_{0} c^{2} \sqrt{1-v^{2} / c^{2}}
$$

and the field kinetic energy:

$$
T_{\|}=\frac{m_{0} v^{2}}{\sqrt{1-v^{2} / c^{2}}}
$$

For the plates moving in the perpendicular direction to the plates' surfaces the situation is similar. The field potential energy will be the same, since the distance between the plates now undergoes the Lorentz length contraction and the area $A=A_{0}$ remains unchanged. The field potential energy for the motion in this direction is thus:

$$
V_{\perp}=\frac{Q^{2} d_{0} \sqrt{1-v^{2} / c^{2}}}{2 \varepsilon_{0} A_{0}}=m_{0} c^{2} \sqrt{1-v^{2} / c^{2}}=V_{\|}
$$

There is no force from the currents and there is no magnetic field in the plates' vicinity now. The field kinetic energy thus cannot be related to the magnetic field in this case, since there is no magnetic field present anywhere. The electric field terminates on charges of the plates, so when the plates move in the perpendicular direction the electric field must follow, but follows with the delays equal to: $t_{ \pm}=d /(c \pm v)$, during which the plates have performed work. The electric field changes linearly within the plates thicknesses, as already mentioned, so the work the top plate has performed and stored in the field originating from the bottom plate is equal to: 


$$
W_{t o p \perp}=\frac{1}{2} F_{q 0} v t_{-}=\frac{1}{2} \frac{Q^{2}}{2 \varepsilon_{0} A_{0}} \frac{v d}{c-v}
$$

calculated similarly as in Equation 6. The bottom plate has returned some of the work back from the field, so the difference is the kinetic field energy when it is assumed that the plates are still maintaining a constant distance $d$ :

$$
T_{\perp}=\frac{1}{2} \frac{Q^{2}}{2 \varepsilon_{0} A_{0}} \frac{v d}{c-v}-\frac{1}{2} \frac{Q^{2}}{2 \varepsilon_{0} A_{0}} \frac{v d}{c+v}=\frac{Q^{2} d}{2 \varepsilon_{0} A_{0}} \frac{v^{2}}{c^{2}-v^{2}}=\frac{m_{0} v^{2}}{\sqrt{1-v^{2} / c^{2}}}=T_{\|}
$$

Since the plates do not perform work in the field originating from charge of the plates themselves, additional factor of $1 / 2$ had to be included in Equation 17 and Equation 18. More details of the derivation of this formula are given in the appendix. Equation 18 is interesting from the point of view of the origin of inertial motion, which seems to be related to the propagation velocity of the trapped EM field energy. The details of this relationship, however, will not be addressed here any further. This is left for the future work. A good review of studies related to the parallel plate capacitors can be found in Janssen (2008).

The EM field energy for the plates moving in either direction is thus the same as expected. This is a well known classical result with no surprise, since this also follows from the fact that the field energy must be a scalar quantity.

$$
W_{e m \|}=W_{e m \perp}=\frac{m_{0} c^{2}}{\sqrt{1-v^{2} / c^{2}}}
$$

\section{The Gravitomagnetic Analogy}

The simplest way to derive the gravitomagnetic analogy for the above described experiment is to compare the Maxwell's field equations with the field equations for the GEM approach according to Rugierro at al. (2002).

$$
\begin{array}{ll}
\nabla \cdot \vec{E}_{g}=-4 \pi \kappa \rho_{g} & \nabla \cdot \vec{E}=\frac{\rho}{\varepsilon_{0}} \\
\nabla \cdot \vec{B}_{g}=0 & \nabla \cdot \vec{B}=0 \\
\nabla \times \vec{E}_{g}=-\frac{1}{2 c} \frac{\partial \vec{B}_{g}}{\partial t} & \nabla \times \vec{E}=-\frac{\partial \vec{B}}{\partial t} \\
\nabla \times \frac{1}{2 c} \vec{B}_{g}=\frac{1}{c^{2}} \frac{\partial \vec{E}_{g}}{\partial t}-\frac{4 \pi \kappa}{c^{2}} \vec{j}_{g} & \nabla \times \vec{B}=\frac{1}{c^{2}} \frac{\partial \vec{E}}{\partial t}+\mu_{0} \vec{j} \\
\vec{F}_{g}=M\left(\vec{E}_{g}+\vec{v} \times \frac{1}{2 c} \vec{B}_{g}\right) & \vec{F}=Q(\vec{E}+\vec{v} \times \vec{B})
\end{array}
$$

The test setup will be the same as for the EM case except that the plates will now be massive and not charged. The plates will have the rest mass $M_{0}$. The gravitoelectric and the gravitomagnetic fields and from them the forces acting between the plates will be obtained similarly as for the electric and magnetic fields from the integral formulas. First, for the stationary plates from the Gauss law it is:

$$
F_{g 0}=\frac{2 \pi \kappa M_{0}^{2}}{A_{0}}
$$

In the next step, similarly as in Equation 2, the gravitomagnetic induction is calculated as follows:

$$
\frac{1}{2 c} B_{g}=\frac{4 \pi \kappa M}{A} \frac{v}{c^{2}}
$$

The formula for the gravitational force between the moving plates is then obtained using Equation 24 considering again the Lorentz length contraction and now also considering the inertial mass increase with velocity. The result is:

$$
F_{g}=\frac{2 \pi \kappa M_{0}^{2}}{A_{0} \sqrt{1-v^{2} / c^{2}}} \frac{\left(1-2 v^{2} / c^{2}\right)}{\left(1-v^{2} / c^{2}\right)}
$$


The equivalent expressions for the gravitational field potential energy is:

$$
V_{g \|}=\frac{m_{g 0} c^{2}\left(1-2 v^{2} / c^{2}\right)}{\left(1-v^{2} / c^{2}\right)^{3 / 2}}
$$

The factor of two in the numerator parenthesis results from the factor of one half standing by the gravitomagnetic induction vector $B_{g}$ of the Lorentz force equation, Equation 24. Similarly, as derived in Equation 12, the equivalent expression for the gravitational field kinetic energy is:

$$
T_{g \|}=\frac{1}{2} \frac{c^{2}}{4 \pi \kappa}\left(\frac{1}{2 c} B_{g}\right)^{2} d_{0} A=\frac{2 \pi \kappa M^{2} d_{0} v^{2}}{c^{2} A}=\frac{m_{g 0} v^{2}}{\left(1-v^{2} / c^{2}\right)^{3 / 2}}
$$

The formulas for the gravitational field energies were again simplified using the abbreviation:

$$
m_{g 0} c^{2}=\frac{2 \pi \kappa M_{0}^{2} d_{0}}{A_{0}}
$$

The total GEM field energy is thus equal to:

$$
W_{g e m \|}=V_{g \|}+T_{g \|}=\frac{m_{g 0} c^{2}\left(1-2 v^{2} / c^{2}\right)}{\left(1-v^{2} / c^{2}\right)^{3 / 2}}+\frac{m_{g 0} v^{2}}{\left(1-v^{2} / c^{2}\right)^{3 / 2}}=\frac{m_{g 0} c^{2}}{\sqrt{1-v^{2} / c^{2}}}
$$

which is seemingly a reasonable result that is expected, similar to the EM case in Equation 19. This result also confirms the correctness of the factor of two in the numerator parenthesis of formulas in Equation 27 and Equation 28.

When the plates' motion is in the perpendicular direction to the plates' surfaces the gravitational field potential energy is:

$$
V_{g \perp}=\frac{2 \pi \kappa M_{0}^{2} d_{0} \sqrt{1-v^{2} / c^{2}}}{A_{0}\left(1-v^{2} / c^{2}\right)}=\frac{m_{g 0} c^{2}}{\sqrt{1-v^{2} / c^{2}}}
$$

If the calculation could stop here that would be wonderful, however, following the same reasoning as for the EM case, Equation 18, there is a gravitational field kinetic energy for the motion in the perpendicular direction, which is:

$$
T_{g \perp}=\frac{2 \pi \kappa M_{0}^{2} d_{0} \sqrt{1-v^{2} / c^{2}}}{A_{0}\left(1-v^{2} / c^{2}\right)} \frac{v^{2}}{c^{2}-v^{2}}=\frac{m_{g} v^{2}}{\left(1-v^{2} / c^{2}\right)^{3 / 2}}=T_{g \|}
$$

The total GEM field energy for the plates' motion in the perpendicular direction is thus equal to:

$$
W_{g e m \perp}=V_{g \perp}+T_{g \perp}=\frac{m_{g 0} c^{2}}{\sqrt{1-v^{2} / c^{2}}}+\frac{m_{g 0} v^{2}}{\left(1-v^{2} / c^{2}\right)^{3 / 2}}=\frac{m_{g 0} c^{2}}{\left(1-v^{2} / c^{2}\right)^{3 / 2}}
$$

The total GEM field energy now surprisingly depends on the motion direction of the plates. This is not acceptable!!

$$
W_{g e m \|}=W_{g e m \perp}\left(1-v^{2} / c^{2}\right)
$$

This is not reasonable and it is a fatal problem for the theory that can be traced back to the relativistic mass dependency on velocity and to the Einstein weak equivalence principle (WEP) where the gravitational mass and the inertial mass have always identical dependencies on velocity. In the Maxwell's EM field theory charge is an absolute invariant independent of velocity while in the Einstein's relativity the mass is variable. This problem thus questions the correctness of the whole concept of the gravitomagnetic force and consequently the Einstein field equations from which the GEM field equations are uniquely and rigorously derived.

The author is somewhat perplexed that the experts working in this field for their entire lives would not know about this problem and continue to use the idea of gravitomagnetic force to solve the various problems of gravitation field, NASA (2004). This gives an impression that the criticism is being purposely avoided and covered up in order to publish more papers on the GEM field theory and secure more funding for such a work. 


\section{Solution of the Problem}

This problem can be resolved by considering that there is no gravitomagnetic force and that the inertial mass and the gravitational mass of moving bodies depend on velocity differently as derived previously by Hynecek (2005).

$$
\begin{gathered}
M_{i}=\frac{M_{0}}{\sqrt{1-v^{2} / c^{2}}} \\
M_{g}=M_{0} \sqrt{1-v^{2} / c^{2}}
\end{gathered}
$$

The gravitational mass dependency on velocity is consistent with the fact that photons, that move at the velocity $c$, have only the inertial mass and do not have any gravitational mass, Okun', Selivanov, and Telegdi (1999). Contrary to the popular belief, the photons do not fall in a gravitational field and gain energy from the fall. The same will be true for the gravitons when their existence is actually proven by observations or experiments.

For the gravitational field potential energy of the plates moving in the parallel direction to their surfaces it is thus:

$$
V_{g \|}=\frac{2 \pi \kappa M_{0}^{2} d_{0}\left(1-v^{2} / c^{2}\right)}{A_{0} \sqrt{1-v^{2} / c^{2}}}=m_{g 0} c^{2} \sqrt{1-v^{2} / c^{2}}
$$

Since there is no gravitomagnetic field and the corresponding gravitomagnetic field energy for this motion direction, there is no gravitational field kinetic energy:

$$
T_{g \|}=0
$$

This indicates that in this direction the gravitational field does not move with the plates. The gravitational field is thus a longitudinal deformation of space in this case in the direction of the gravitational force, perpendicular to the plates' surfaces. However, the space deformation is not occurring between the plates, it occurs only in the space outside of the plates, therefore, this deformation does not have to be considered for the calculation of the field energy. The total field energy for this motion direction is thus equal to:

$$
W_{g \|}=V_{g \|}+T_{g \|}=m_{g 0} c^{2} \sqrt{1-v^{2} / c^{2}}
$$

For the plates moving in the perpendicular direction to their surfaces the field potential energy is:

$$
V_{g \perp}=\frac{2 \pi \kappa M_{0}^{2} d_{0}\left(1-v^{2} / c^{2}\right)^{3 / 2}}{A_{0}}=m_{g 0} c^{2}\left(1-v^{2} / c^{2}\right)^{3 / 2}
$$

The gravitational field kinetic energy, following the similar derivation as shown in Equation 33, and assuming again that the gravitational field is propagating with the velocity $c$, is equal to:

$$
T_{g \perp}=\frac{2 \pi \kappa M_{0}^{2} d_{0}\left(1-v^{2} / c^{2}\right) \sqrt{1-v^{2} / c^{2}}}{A_{0}} \frac{v^{2}}{c^{2}-v^{2}}=m_{g 0} v^{2} \sqrt{1-v^{2} / c^{2}}
$$

The total gravitational field energy for this motion direction is thus:

$$
W_{g \perp}=m_{g 0} c^{2}\left(1-v^{2} / c^{2}\right)^{3 / 2}+m_{g 0} v^{2} \sqrt{1-v^{2} / c^{2}}=m_{g 0} c^{2} \sqrt{1-v^{2} / c^{2}}
$$

The energy stored in the field is again independent of the direction of motion as is expected and as it should be:

$$
W_{g \perp}=W_{g \|}=m_{g 0} c^{2} \sqrt{1-v^{2} / c^{2}}
$$

This simple fact confirms once more the gravitation mass dependency on velocity as derived again by Hynecek (2009) and invalidates the gravitomagnetic force concept.

Of course, there is also the energy associated with the inertial and gravitational masses of charge and the inertial mass of the plates themselves, which is equal to: 


$$
W_{i}=\frac{2 M_{0} c^{2}}{\sqrt{1-v^{2} / c^{2}}}
$$

This was for simplicity omitted from considerations, since it is not necessary for the proof of nonexistence of the gravitomagnetic force.

\section{Conclusions}

In this article it was clearly shown, based on simple principles and a simple model, that there is no gravitomagnetic force and no gravitomagnetic field. The fundamental reason for this fact is the nature of the gravitational force, which has an unipolar character with only attractive forces between the massive bodies with the mass depending on velocity. The carriers of this force are probably the spin zero gravitons, which suggest that the gravitational field most likely propagates as longitudinal compression waves with a velocity $c$. The article has also clearly shown that the gravitation field energy depends on velocity as:

$$
W_{g}=m_{g 0} c^{2} \sqrt{1-v^{2} / c^{2}}
$$

which is the second important reason for the nonexistence of the gravitomagnetic force.

This clearly contrasts with the EM field that is bipolar generated by both the positively and negatively charged particles with charge being an universal invariant. The force carriers are the unity spin photons that, as is well known, propagate as transversal waves. The EM field energy depends on velocity differently than the gravitational field energy as:

$$
W_{e m}=m_{0} c^{2} / \sqrt{1-v^{2} / c^{2}}
$$

It is, therefore, misleading to draw the analogy for the gravitational field from the Maxwell's EM field theory and thus naively expect that there is a gravitational analog to a magnetic field.

Finally, the paper also concludes that Einstein field equations of general relativity may not be correct, since the GEM field equations and the equation for the gravitomagnetic force are rigorously derived from them.

While there are very many enthusiasts supporting the gravitomagnetism it is not often mentioned that there is also some controversy about it as can be found in Wikipedia (http://en.wikipedia.org/wiki/Gravitoelectromagnetism) and in publication citations (ECD, http://www.osti.gov/energycitations/product.biblio.jsp?osti_id=21421194).

\section{References}

Clark, S. J., \& Tucker, R. W. (2000). Gauge Symmetry and Gravito-Electromagnetism. Retrieved from http://arxiv.org/abs/gr-qc/0003115v2

Hynecek, J. (2005). Remarks on the Equivalence of Inertial and Gravitational Masses and on the Accuracy of Einstein's Theory of Gravity. Phys. Essays, 18, 182.

Hynecek, J. (2009). The Galileo Effect and the General Relativity Theory. Phys. Essays, $22,551$.

Janssen, Michel H. P. (2008). Drawing the line between kinematics and dynamics in special relativity. Symposium on Time and Relativity.

Mashhoon, B., Gronwald, F., \& Lichtenegger, I. M. (1999). Gravitomagnetism and the Clock Effect. Retrieved from http://arxiv.org/abs/gr-qc/9912027v1

NASA. (2004). In Search of Gravitomagnetism Gravity Probe B has left Earth to measure a subtle yet long-sought force of Nature. http://science.nasa.gov/science-news/science-at-nasa/2004/19apr_gravitomagnetism/

Okun', L. B., Selivanov, K. G., \& Telegdi, V. L. (1999). Gravitation, photon, clocks. Physics-Uspekhi, 42(10).

Ruggiero, M. L., \& Tartaglia, A. (2002). Gravitomagnetic Effects. Retrieved from http://arxiv.org/abs/gr-qc/ $0207065 \mathrm{v} 2$ 


\section{Appendix}

The derivation of the formula for the kinetic energy stored in the field of the two parallel plates moving in the direction perpendicular to their surfaces, Equation 18, can be best understood from the potential diagram in Figure 2. This diagram illustrates the potentials between the plates that move in the $z$ direction with a constant velocity $v$ and includes the retarded positions of the plates. For the electric field intensity that is obtained as a gradient of the potential then holds that:

$$
E=-\partial \varphi / \partial z
$$

The field intensity is then calculated according to the Gauss law:

$$
\begin{gathered}
\oiint \vec{D} \cdot d \vec{S}=Q \\
|E|=\frac{Q}{2 \varepsilon_{0} A_{0}}-\frac{-Q}{2 \varepsilon_{0} A_{0}}=\frac{Q}{\varepsilon_{0} A_{0}}
\end{gathered}
$$

Assuming that the field propagates with the speed of light $c$, the time it takes for the field of the bottom plate to reach the top plate across the distance corresponding to the retarded position of the bottom plate: $d+v t_{-}=c t_{-}$is:

$$
t_{-}=d /(c-v)
$$

The work that the top plate has performed in the previous field intensity of the bottom plate before the field from charge at the new position of the plate has reached it is:

$$
W_{-}=F_{-} v t_{-}=\frac{1}{2} \frac{Q^{2}}{2 \varepsilon_{0} A_{0}} v t_{-}=\frac{1}{2} \frac{Q^{2} v}{2 \varepsilon_{0} A_{0}} \frac{d}{c-v}
$$

Here it is important to realize that the plates do not perform work in the field of their own charge and that the plates' thicknesses are: $z_{\mathrm{p}}<<d$. This accounts for the additional factor of 1/2 in Equation A5. Similarly, for the bottom plate that is being pulled up and receives the work from the field it is:

$$
W_{+}=F_{+} v t_{+}=\frac{1}{2} \frac{Q^{2}}{2 \varepsilon_{0} A_{0}} v t_{+}=\frac{1}{2} \frac{Q^{2} v}{2 \varepsilon_{0} A_{0}} \frac{d}{c+v}
$$

This is because the retardation time of the field propagation from the top plate to the bottom plate is:

$$
t_{+}=d /(c+v)
$$

The difference between the work of the top plate delivered to the field and the work returned from the field to the bottom plate is proportional to the potential difference: $T_{\perp} \approx \varphi_{-}-\varphi_{+}$, and is equal to the field kinetic energy:

$$
T_{\perp}=F_{-} v t_{-}-F_{+} v t_{+}=\frac{1}{2} \frac{Q^{2} d}{2 \varepsilon_{0} A_{0}}\left(\frac{v}{c-v}-\frac{v}{c+v}\right)=\frac{Q^{2} d}{2 \varepsilon_{0} A_{0}} \frac{v^{2}}{c^{2}-v^{2}}=\frac{m_{0} v^{2}}{\sqrt{1-v^{2} / c^{2}}}
$$

In the final expression for the kinetic field energy the Lorentz length contraction formula: $d=d_{0}\left(1-v^{2} / c^{2}\right)^{1 / 2}$ and the following substitution for the rest mass of the field were used:

$$
\frac{Q^{2} d_{0}}{2 \varepsilon_{0} c^{2} A_{0}}=\frac{1}{2} \mu_{0} \frac{Q^{2} d_{0}}{A_{0}}=m_{0}
$$

The motion is uniform, therefore, this difference does not change and it remains one of the constants of motion.

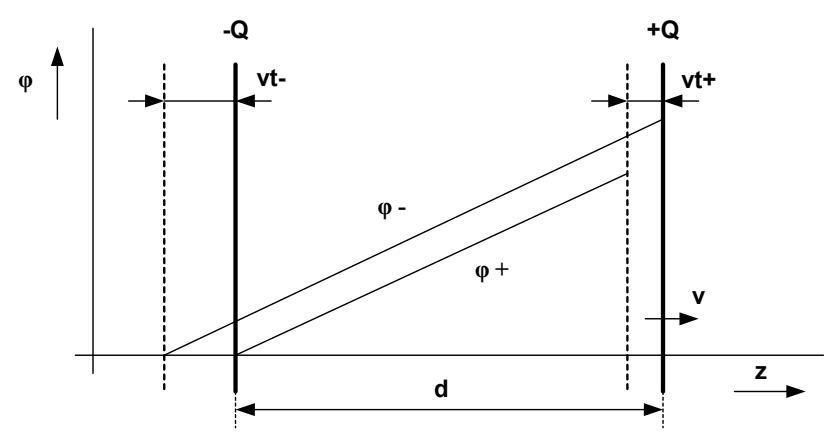

Figure 2. Potential diagram and the retarded plate positions of charged parallel plates moving in the direction perpendicular to their surfaces. The potential difference: $\varphi_{-}-\varphi_{+}$remains constant as the plates move 